\title{
ENIGMA, LES DÉCRYPTEURS POLONAIS ET LES SERVICES SECRETS FRANÇAIS, 1932-1945
}

Jean-Charles Foucrier http://orcid.org/0000-0002-5623-571X

Université Paris-Sorbonne - Paris IV

\section{ABSTRACT \\ ENIGMA, THE POLISH CODEBREAKERS AND THE FRENCH SECRET SERVICE, 1932-1945}

In the early 1930's, the Polish codebreakers succeeded while all the others failed: they broke the Enigma. Three young and brilliant mathematicians, Marian Rejewski, Henryk Zygalski and Jerzy Różycki managed to read the German cyphertexts from 1933 to as late as 1939. But this huge success remained a secret for a long time, unknown in France and England. After the fall of Poland in September 1939, the three mathematicians linked their fate with the French secret service and kept breaking the Enigma code. Again, following the French defeat of June 1940, they experienced exile and irremediably sank into oblivion. Today, the story of the Polish codebreakers and the French secret service remains very little known in France, although their work proved decisive in the Allied victory during the Second World War.

Key words: World War II, Enigma, codebreaking, secret service.

Słowa kluczowe: II wojna światowa, Enigma, łamanie szyfru, tajne służby.

Août 1932. Trois jeunes Polonais s'engouffrent dans le vaste Palais Saxon de Varsovie (Pałac Saski), siège du Bureau du Chiffre (Biuro Szyfrów). Marian Rejewski, Henryk Zygalski et Jerzy Różycki représentent l'élite des mathématiciens polonais, fraîchement diplômés de l'Université Adam Mickiewicz de Poznań. Ils viennent de délaisser des carrières universitaires prometteuses pour se consacrer pleinement au service de la nation, à travers le décryptage des codes étrangers. Rejewski, Zygalski et Różycki vont en quelques mois réussir l'impossible, déchiffrer Enigma. Ils vont également se retrouver ballotés au fil des intrigues de la diplomatie et des services secrets alliés, pour finalement sombrer dans l'oubli. 


\section{L'EXPLOIT DES MATHÉMATICIENS POLONAIS}

Le Bureau du Chiffre polonais dispose en 1932 d'une solide expérience en matière de décryptage. Après avoir recouvré son indépendance en 1918, la jeune Deuxième République s'est légitimement sentie oppressée par ses ombrageux voisins russes et allemands. La nécessité de disposer d'un service de décryptage s'est rapidement fait sentir. Durant la guerre russo-polonaise (1919-1921), sous la férule du lieutenant-colonel Jan Kowalewski une équipe de mathématiciens civils est parvenue à briser les codes soviétiques, intervenant de manière décisive en 1920 au cours de la bataille de Varsovie ${ }^{1}$.

Dix années plus tard, le Bureau du Chiffre polonais se heurte cette fois-ci à une nouvelle machine à coder allemande, Enigma, librement disponible sur le marché civil. Utilisée par l'armée allemande dans une version modifiée, Enigma pose des problèmes insolubles au chef du Bureau du Chiffre polonais, le major Gwido Langer, et à son adjoint et chef de la section allemande «BS-4 », le major Franciszek Pokorny. Avec une possibilité de combinaisons estimée à un total astronomique de $5 \times 10^{87}$, Enigma semble absolument indécodable 2 . Les deux chefs polonais finissent par organiser un premier cours de cryptologie à destination des officiers en 1928, afin de mettre la main sur une équipe de cerveaux capables de vaincre Enigma. Face aux médiocres résultats enregistrés, il apparaît toutefois très vite que ces derniers ne proviendront pas de l'armée. Comme en 1920, les espoirs reposent une nouvelle fois sur des mathématiciens d'origine civile.

Une nouvelle série de cours de cryptologie est organisée à l'Université de Poznań, du 15 janvier au 22 mars 1929, réalisés sur l'initiative du major Franciszek Pokorny et grâce au travail du directeur de l'Institut de mathématiques de l'Université de Poznań, le professeur Zdzisław Krygowski ${ }^{3}$. Vingt-trois jeunes mathématiciens sont sélectionnés, la compréhension parfaite de l'allemand étant un critère primordial - ce qui explique en bonne partie le choix de l'Université de Poznań, ancien territoire allemand. Henryk Zygalski, Jerzy Różycki et surtout Marian Rejewski se distinguent parmi les meilleurs. D'une intelligence supérieure, imaginatifs, intuitifs et énergiques, les trois jeunes Polonais sont secrètement admis dans les derniers mois de 1929 dans les sous-sols du haut-quartier militaire de Poznań, rue Sainte-Marcin ${ }^{4}$.

1 R.J. Hanyok, Before Enigma: Jan Kowalewski and the early days of the Polish Cipher Bureau (1919-1922) [in:] W. Kozaczuk, J. Straszak, Enigma: How the Poles Broke the Nazi Code, New York 2004, p. 87-95.

2 J. Stengers, Enigma, the French, the Poles and the British 1931-1940, Revue belge de philologie et d'histoire, tome 82, fasc. 1-2, 2004, p. 449.

3 Z.J. Kapera, Marian Rejewski, the Man who defeated Enigma, The Enigma Bulletin, No. 8, édité par Z.J. Kapera, Cracovie 2013, p. 76.

4 Marian Rejewski quitte toutefois temporairement l'Université de Poznań en mars 1929 pour la très prestigieuse université allemande de Göttingen, formatrice d'un grand nombre de génies mathématiciens et physiciens dont Max Born, Werner Heisenberg, Victor Weisskopf et Enrico Fermi. Rejewski est de retour à Poznan au cours de l'automne 1930, le professeur Zdzilaw Krygowski lui offrant une charge d'enseignement. 
Après avoir œuvré avec succès à décrypter des codes secondaires utilisés par l'armée allemande, Zygalski, Różycki et Rejewski sont transférés au Bureau du Chiffre Polonais à Varsovie, où Rejewski puis ses deux amis apprennent l'existence d'Enigma et s'attachent à en venir à bout.

En 1930, le major Franciszek Pokorny est remplacé par le capitaine Maksymilian Ciężki à la tête du BS-4. Afin d'apporter une aide à ses nouveaux décrypteurs, Ciężki remet à Rejewski des documents ultra-secrets, incluant les réglages d'Enigma pour les mois de septembre et octobre 1932, sans en révéler l'origine. Ces derniers proviennent dans les faits d'un agent allemand œuvrant secrètement pour les services de renseignements français, Hans-Thilo Schmidt (nom de code Asché). Schmidt travaille au Service du Chiffre de l'armée allemande, et possède ainsi tous les secrets d'Enigma. Opposant au parti nazi (dont il fait néanmoins partie en guise de couverture), Hans-Thilo Schmidt est surtout un bon vivant, aimant les vêtements de luxe, la vie mondaine et les femmes - des centres d'intérêts certes agréables mais pour le moins onéreux. L'Allemand n'hésite donc pas à vendre des documents secrets sur Enigma contre très forte rétribution, généreusement réglée par le gouvernement français par l'intermédiaire du capitaine Gustave Bertrand, chef de la Section D du Service du Chiffre français, chargée de prodiguer le matériel nécessaire pour percer les mystères des procédés cryptographiques étrangers.

Avec les documents fournis par Bertrand et son agent Asché, et également grâce à son intelligence exceptionnelle, Rejewski parvient en janvier 1933 à réussir l'impossible : déchiffrer Enigma ${ }^{5}$. Lui et ses camarades développent par la suite des procédés manuels et mécaniques de décryptage, comme le « cyclomètre » et la «bom$b a »$ de Rejewski, les « Feuilles de Zygalski », ou encore la « méthode de l'horloge » de Różycki. Toutes ces méthodes originales permettent au Bureau du Chiffre polonais de déchiffrer les messages Enigma à peu près tous les jours, et cela jusque dans les premiers mois de l'année $1939^{6}$. Toutefois, le succès des mathématiciens polonais ne dépasse pas les murs épais du Palais Saxon. Car durant toutes ces années, de manière a priori surprenante, les Polonais ne révèlent rien de leur découverte à leurs alliés Français, ni à aucune autre nation.

5 G. Bloch, The French Contribution to the Breaking of Enigma, The Enigma Bulletin, No. 1, édité par Z.J. Kapera, Cracovie, décembre 1990. L'impact décisif des documents fournis par Hans-Thilo Schmidt est généralement admis par les spécialistes d'Enigma, dont Hugh Sebag-Montefiore. D'autres experts comme Wladyslaw Kozaczuk et Zdzilaw Jan Kapera estiment cette aide comme non nécessaire, bien que particulièrement utile. Dans les tous les cas, les documents d'Asché ont assurément facilité le travail des décodeurs polonais, tout en renforçant la coopération entre les services secrets français et polonais.

6 Toutefois à partir de septembre 1938 une série de contre-mesures allemandes limitent drastiquement le décodage d'Enigma, restreint à quelques messages de l'armée et du Sicherheitsdienst (SD - le service de renseignement de la SS). 


\section{LA POLOGNE DANS LE JEU DIPLOMATIQUE INTERNATIONAL DES ANNÉES 30}

Depuis la guerre russo-polonaise en 1920, les services de renseignements français et polonais collaborent dans l'échange d'informations sur la Russie et l'Allemagne. Même après la fin de la mission militaire française à Varsovie, un protocole secret signé le 13 avril 1926 maintient un officier de liaison français dans la capitale polonaise ${ }^{7}$. Cette coopération atteint son sommet au début des années 30 , grâce à l'effort énergique de Gustave Bertrand, qui se rend régulièrement à Varsovie pour rencontrer Langer et son adjoint Ciężki, et ainsi alimenter le Bureau du Chiffre polonais avec les documents fournis par son agent Asché. Mais malgré les relations très cordiales et bienveillantes entre les trois hommes, Bertrand est tenu en dehors des exploits de Rejewski et de ses camarades. Sur la scène internationale, les années 30 sont en effet marquées par la politique du ministre des Affaires étrangères Józef Beck, qui louvoie habilement entre la Russie et l'Allemagne, au détriment toutefois d'une alliance forte avec la France et le Royaume Uni.

Même s'il est loin d'être le germanophile souvent décrié dans l'historiographie, le colonel Beck se méfie puissamment de son voisin soviétique, tout en cherchant à préserver un équilibre avec l'Allemagne. Il souhaite avant tout garder la main sur le destin de la Pologne, déclarant ainsi : « La Pologne est un sujet et non un objet de la politique extérieure ${ }^{8} \gg$. Ce qui se traduit dans les faits par la signature du pacte de non-agression germano-polonais en janvier 1934, un an après l'accession d'Hitler au pouvoir. La France suit une toute autre préférence, son président Édouard Herriot souhaitant intégrer l'URSS dans le concert de la sécurité européenne. Le traité franco-soviétique d'assistance mutuelle de mai 1935 porte un très dur coup aux relations franco-polonaises. Par ailleurs, les services de renseignements polonais sont parfaitement au courant de la faiblesse des codes français, couramment déchiffrés par les Allemands ${ }^{9}$. Le risque serait en conséquence trop grand de voir les Allemands incidemment découvrir la vérité. Les Polonais n'ont aucun intérêt à risquer de révéler leur secret sur Enigma, et ainsi mécontenter leur puissant voisin occidental.

De leur côté, les services de renseignements français s'intéressent aussi à Enigma. Ils ont même eu accès les premiers aux documents de Asché en 1932, mais les ont considérés comme insuffisants - ce qui avait poussé Bertrand à se tourner vers le major Langer et le capitaine Ciężki. Bertrand, qui suspecte à raison les réussites cachées de ses camarades polonais, s'exaspère du manque de volonté des services français. « Si nos spécialistes français avaient bien voulu consentir l'effort intellectuel des

7 O. Forcade, La République secrète : Histoire des services spéciaux français de 1918 à 1939, Paris 2013, p. 270.

8 F. Dessberg, La Russie et l'URSS : un élément dérangeant des relations franco-polonaises au $X X^{e}$ siècle, Prace Historyczne, 142, Université Jagellonne, Cracovie, 2015, p. 611.

9 Hans-Thilo Schmidt en sera lui-même victime en 1943, démasqué par les décodages allemands de l'ambassade française à Berlin, qui révèlent son existence. Arrêté et torturé, Asché se suicide en prison la même année. 
décrypteurs polonais, nous n'en serions pas à quémander leur aide et à attendre les conclusions de leurs travaux ", déclare Bertrand, $\operatorname{amer}^{10}$. Certes, si les décrypteurs français ont obtenu de brillants résultats durant la Première Guerre mondiale, il n'en est plus de même au début des années 30 .

Le chef du service de décryptement français est alors le capitaine Henri Braquenié. Son dossier militaire le décrit comme « intelligent, faisant preuve d'excellentes qualités techniques mais mou et indécis $\rangle^{11}$. Plus grave encore, Braquenié n'est qu'officier de réserve, son métier civil étant ingénieur électricien. Le Français souffre ainsi pour le moins de la comparaison avec les jeunes génies Polonais, bardés de diplômes universitaires en mathématiques pures. Même si ses supérieurs déclarent avec une satisfaction douteuse en 1940 que le capitaine Braquenié " a dirigé avec beaucoup de tact et de compétence une équipe spéciale qui a produit des résultats $»^{12}$, les Français sont bien incapables de décrypter par eux-mêmes Enigma. De leur côté, les Britanniques ne s'intéressent que tardivement au sujet, après l'arrivée des nazis au pouvoir. Disposant comme les Polonais des meilleurs universitaires, leurs services de décryptement débutent toutefois avec un retard conséquent, et se montrent eux-aussi incapables d'exploiter les documents de Asché. Dilly Knox, le brillant meneur des décrypteurs britanniques, parvient seulement à décoder une version « $\mathrm{K} » \mathrm{~d}^{\prime}$ 'Enigma, utilisée par les nationalistes durant la guerre d'Espagne, mais échoue face à l'Enigma allemande.

La situation évolue à la fin de 1938. Les mesures prises par le Service du Chiffre allemand pour compliquer la sécurité d'Enigma portent leurs fruits. Les Polonais ont désormais toutes les difficultés pour décrypter. En parallèle, la menace nazie sur la Pologne devient évidente. Une première conférence à Paris les 9 et 10 janvier 1939 permet enfin de réunir à la même table Langer, Ciężki, Knox, Bertrand, Braquenié et Alastair Denniston, le chef du Service de Décryptement britannique. Les protagonistes, méfiants, campent sur leurs positions. On se sépare sur une évasive promesse : «Toutes les questions ayant pour but d'attaquer les moyens de chiffrement en vigueur et, par suite, la recherche du renseignement dans ce domaine, seront envisagées en commun $»^{13}$.

Cette perspective voit le jour les 26 et 27 juillet suivants près de Pyry, à 20 kilomètres de Varsovie, où dans les bois sombres de Kabackie les Polonais ont installé leur nouveau QG du Bureau du Chiffre. Organisée par Langer et Ciężki, la conférence se veut cette fois-ci détendue de la part des Polonais, à la surprise de leurs invités français et britanniques, incluant Denniston, Knox, Bertrand et Braquenié. Face à une assistance médusée, le capitaine Ciężki révèle posément le grand secret des décrypteurs polonais, qui déchiffrent depuis maintenant plusieurs années Enigma. Dilly Knox, exaspéré par la compétence limitée de Ciężki en matière de décryptage

10 P. Paillole, Notre espion chez Hitler, Paris 2013, p. 58.

${ }_{11}$ Service Historique de la Défense de Vincennes (SHD), AI 1 P 20377 1, Dossier militaire d'Henri Braquenié.

12 Ibidem.

13 SHD, DE 2016 ZB 25, Fonds G. Bertrand, Étude des possibilités de décryptement des interceptions radios allemandes chiffrés avec la machine « ENIGMA » de l'Armée, 14 janvier 1939. 
et surtout mortifié dans sa fierté toute britannique, ne dit rien pendant de longues heures, puis finit par exploser le soir, comme le rapporte son chef Denniston : "Toute l'affaire est une question d'espionnage, ne cessait-il de répéter. Ils n'ont jamais rien fait, ils ont eu recours à un espion et ont ainsi été en mesure de décoder, comme n'importe qui aurait pu le faire $!^{14} »$. Le lendemain 27 juillet, Knox, beaucoup plus calme, rencontre Zygalski, Różycki et Rejewski. Ces derniers, enchantés d'enfin découvrir leurs homologues britanniques et français, expliquent amicalement le fonctionnement de leurs bomby et des Feuilles de Zygalski ${ }^{15}$. Knox reconnaît aussitôt l'intelligence et la compétence des jeunes mathématiciens, et admet l'avance réalisée par les Polonais : « Je suis certain que Ciężki en sait fort peu au sujet de la machine, déclaret-il peu après à Denniston. En revanche, les jeunes hommes semblent très capables et honnêtes ${ }^{16}$ ». Les participants se séparent finalement sur de chaudes promesses de collaboration, et d'envois de bomby, de Feuilles de Zygalski et de reproductions de machines Enigma à Paris et Londres. Des actes aussi concrets que tardifs, un mois avant le traité de non-agression germano-soviétique. Quelques jours plus tard, les chars allemands écrasent de leur chenille les postes frontières polonais, et débutent la Seconde Guerre mondiale. La Pologne sombre en trois semaines.

\section{QUATRE EXILS FORCÉS, ET L'OUBLI EN RÉCOMPENSE}

Les décrypteurs polonais sont évacués de Varsovie bombardée, contraints de laisser leur famille et de passer en Roumanie, qu'ils atteignent en voiture et en bus dans la nuit du 17 septembre 1939, alors que se déclenche l'agression russe. Ils se rendent en train à Bucarest, où l'ambassadeur britannique leur explique qu'il ne saurait prendre de décision sans l'avis de Londres. Impatients, les Polonais tentent leur chance à l'ambassade française, où de manière moins protocolaire, on leur fourni aussitôt visas et billets pour Paris. En octobre, les décodeurs sont à l'abri en France, réunis au château de Vignolles à 40 kilomètres au nord-est de Paris, dans le clandestin « PC Bruno », sous l'égide de Bertrand. Leurs chefs Langer et Ciężki les rejoignent par la suite, extirpés de leurs camps de réfugiés roumains où ils stagnaient ${ }^{17}$. En parallèle, à Bletchley Park en Angleterre, les décrypteurs britanniques exploitent efficacement les Feuilles de Zygalski, dont la production complète est terminée en décembre 1939. Le 17 janvier 1940, le PC Bruno est en mesure de décoder à nouveau Enigma, suivi peu après par Bletchley Park le 22 janvier $^{18}$. Bertrand est de plus en plus désabusé

14 R. Erskine, The Poles Reveal their Secrets: Alastair Denniston's Account of the July 1939 Meeting at Pyry, Cryptologia, 30 (4), 2006, p. 300.

15 Braquenié, nullement professionnel du décryptage ni des mathématiques pures, a bien du mal à comprendre les explications de Rejewski, confirmant l'incapacité des services français à décoder Enigma. «Il n'était pas très fort », déclare ainsi diplomatiquement Rejewski. J. Stengers, op.cit., p. 464.

16 M. Batey, Dilly Knox, The Man who broke Enigmas, Londres 2009, p. 76.

17 H. Sebag-Montefiore, Enigma: The Battle for the Code, Londres 2000, p. 55-59.

18 Z.J. Kapera, The Triumph of Zygalski's sheets, Cracovie 2015, p. 93-94. 
face à l'incompétence manifeste des services secrets français, tout comme son camarade le commandant Paul Paillole, l'un des chefs du contre-espionnage français : «Faute de cryptologues compétents, Bertrand n'a pas les moyens de pourvoir à leur réglage quotidien, donc de les faire parler. Ce qu'il appelle la carence de la section du Chiffre de l'État-Major de l'armée l'oblige à rechercher à nouveau l'assistance des savants britanniques et polonais. Une fois encore ce sont ces derniers qui viendront à son secours et cela dans des circonstances dramatiques $»^{19}$. En effet, les décrypteurs polonais réussissent une nouvelle fois l'exploit de décrypter 3842 messages ennemis jusqu'en juin 1940, avertissant notamment de l'imminence de l'attaque allemande à l'ouest - sans réaction de la part de l'état-major français, qui repousse par ailleurs une proposition de Londres de fusionner tous les services de décryptage alliés ${ }^{20}$.

Le 10 mai 1940, les Polonais subissent leur deuxième exil forcé face à l'offensive allemande à l'Ouest qu'ils avaient pourtant anticipée, pour se retrouver cette fois-ci au 2 bis avenue de Tourville à Paris. Le 10 juin, les Polonais entament leur troisième exil, ponctué de pauses à la Ferté-Saint-Aubain, Vensat, puis Toulouse, où ils apprennent la capitulation de la France le 22 juin $^{21}$. Dès le mois de décembre 1940, l'énergique Bertrand reconstitue un «PC Cadix » au château des Fouzes près d'Uzès et du Pont du Gard, où Rejewski, Zygalski, Różycki et quatorze autres Polonais reprennent leurs travaux de décryptage. Le décodage s'effectue en « zone libre », dans le sud de la France non occupée par l'Allemagne, mais dirigée par le gouvernement collaborateur du maréchal Philippe Pétain. Les décrypteurs travaillent en étroite collaboration avec les services secrets polonais et britanniques en Angleterre.

Au début de 1941, une antenne du «PC Cadix » est ouverte en Afrique du Nord, « P.O.1 Kouba » à Alger, dirigée par Ciężki, qui supervise une équipe d'une demi-douzaine de décodeurs alternant avec le « PC Cadix» tous les trois ou quatre mois. C'est au cours d'une de ces traversées de la Méditerranée que Jerzy Różycki disparait tragiquement le 9 janvier 1941, victime d'un naufrage accidentel. Au cours de l'été suivant, les signes d'une invasion de la zone libre se multiplient. Le PC Cadix est évacué le 9 novembre, deux jours avant l'attaque nazie. En deux ans, la station n'a cessé de monter en puissance, interceptant un total de 13000 messages ennemis ${ }^{22}$. De nombreuses informations cruciales ont été transmises aux Alliés, notamment sur les opérations du SD et l'organisation de la Luftwaffe dans le sud de la France, les exactions des SS dans les pays de l'Est, la situation ennemie en Afrique du Nord en prévision du débarquement allié, ou encore l'état de faiblesse des codes chiffrés suisses $^{23}$.

Suite à l'invasion allemande du sud de la France le 11 novembre 1942, les Polonais subissent leur quatrième exil forcé en trois ans. Plus ou moins aidés par la Résistance française souvent mal organisée, les décrypteurs polonais enchaînent de

19 P. Paillole, op. cit., p. 187.

20 G. Bertrand, Enigma, ou la plus grande Enigme de la guerre, Paris 1972, p. 79.

${ }_{21}$ W. Kozaczuk, J. Straszak, Enigma : How the Poles Broke the Nazi Code, New York 2004, p. $35-41$.

22 J. Medrala, Les réseaux de renseignements franco-polonais, Paris 2005, p. 184-185.

23 Z.J. Kapera, In the Shadow of Pont du Gard, Cracovie 2011, p. 96-97. 
multiples aventures rocambolesques en territoire occupé, tentant de franchir les Pyrénées au péril de leur vie. Les deux jeunes mathématiciens y parviennent en janvier 1943, mais Langer et son adjoint Ciężki sont arrêtés en mars, et vont passer le restant de la guerre en camp de prisonniers en Allemagne. Les Polonais n'ont à cette date plus rien à attendre des services de renseignements français, désormais déchirés dans une guerre fratricide entre le Bureau Central de Renseignements et d'Action (BCRA) du général de Gaulle à Londres, et les Services de Renseignement (SR) du général Giraud en Afrique du Nord ${ }^{24}$.

A peu près démunis de tout, emprisonnés plusieurs semaines en Espagne, les survivants polonais parviennent péniblement en Angleterre en août 1943. Mais les services de décryptements britanniques ont bien changé depuis 1939. Alastair Denniston a été écarté sans ménagement en février 1942, et Dilly Knox vient de mourir en février 1943, rongé par un cancer et épuisé dans son combat contre Enigma. Ce sont maintenant des noms inconnus des jeunes Polonais qui font la loi à Bletchley Park : Edward Travis, Gordon Welchman, et un certain Alan Turing. Rendus suspects après avoir passé deux ans en France occupée, et eux-aussi quasiment inconnus à Bletchley Park, Rejewski et Zygalski se voient refuser d'aider les décrypteurs britanniques, et rejoignent le bataillon radio du commandement en chef polonais à Stanmore-Boxmoore, dirigé par le major Tadeusz Lisicki. Ils passent le restant de la guerre à décrypter avec succès les codes secondaires du SD et de la SS, et également quelques codes des alliés soviétiques.

Les deux mathématiciens terminent la guerre sans gloire en mai 1945. Zygalski reste en Angleterre, enseignant à l'université de Surrey. Il reçoit de justesse un doctorat honoris causa de l'Université polonaise en exil en 1977, puis meurt dans l'anonymat le plus complet en 1978. Rejewski rentre en Pologne dans sa ville natale de Bydgoszsz en 1946, mais se heurte au même accueil glacial, cette fois-ci de la part des communistes polonais. Écarté des postes académiques, le vainqueur d'Enigma reçoit de justesse en 1978 la décoration Polonia Restituta, deux ans avant sa mort. La plupart des décrypteurs britanniques ne découvrent l'existence de leurs confrères polonais qu'après leur mort. Gordon Welchman, le grand architecte de Bletchley Park, est stupéfait d'apprendre en 1981 l'histoire de Rejewski, Zygalski et Różycki, dont les travaux ont été « un cadeau d'immense valeur » pour les Britanniques ${ }^{25}$. Alan Stripp, un autre décrypteur britannique, se confond lui-aussi en hommages rétrospectifs : « Leurs travaux ont joué un rôle important en aidant le Royaume-Uni à survivre lors des premières années de la guerre, et à obtenir la victoire finale six années plus $\operatorname{tard}^{26}{ }^{2}$.

Enfin, en 2014 avec le film The Immitation Game, le grand public découvre massivement l'existence d'Enigma et d'Alan Turing, lui-même victime fatale du secret et de l'ignorance ${ }^{27}$. Une juste reconnaissance, certes tardive pour le génie britannique.

24 L. Rivet, Carnets du chef des services secrets, 1936-1944, annotés par O. Forcade et S. Laurent, Paris 2010, p. 544-567.

25 G. Welchman, The Hut Six story: Breaking the enigma codes, Londres 1997, p. 13.

26 A. Stripp, A British salutes the Polish cryptanalysts [in :] W. Kozaczuk, J. Stras zak, Enigma: How the Poles Broke the Nazi Code, New York 2004, p. 123.

27 M. Tyldum, The Imitation Game, 2014. 
Hélas, l'histoire se répète : aucune mention n'est faite des exploits décisifs et des aventures dramatiques des décrypteurs polonais.

\section{BIBLIOGRAPHIE}

\section{Sources}

Service Historique de la Défense (SHD) de Vincennes :

Fond Gustave Bertrand : dossier DE 2016 ZB 25, boîtes 4 \& 5.

Personnel de l'Armée de l'Air : capitaine Henri Braquenié, dossier AI 1 P 203771.

\section{Publications}

Batey M., Dilly Knox, the Man who broke Enigmas, Londres 2009.

Bertrand G., Enigma, ou la plus grande Enigme de la guerre, Paris 1972.

Bloch G., The French Contribution to the Breaking of Enigma, The Enigma Bulletin, No. 1, édité par Z.J. Kapera, Cracovie, décembre 1990.

Erskine R., The Poles Reveal their Secrets : Alastair Denniston's Account of the July 1939 Meeting at Pyry, Cryptologia, 30 (4), 2006, p. 294-305.

Dessberg F., La Russie (et l'URSS : Un élément dérangeant des relations franco-polonaises au XX' siècle, Prace Historyczne, 142, Université Jagellonne, Cracovie, 2015, p. 605-620.

Forcade O., La République secrète : Histoire des services spéciaux français de 1918 à 1939, Paris 2013.

Hanyok R.J., Before Enigma: Jan Kowalewski and the early days of the Polish Cipher Bureau (1919-1922) [in :] W. Kozaczuk, J. Straszak, Enigma: How the Poles Broke the Nazi Code, Hippocrene Books, 2004, p. 87-95.

Kapera Z., Marian Rejewski, the Man who defeated Enigma, Cracovie 2013.

Kapera Z., The Triumph of Zygalski's sheets, Cracovie 2015.

Kapera Z., In the Shadow of Pont du Gard, Cracovie 2011.

Kozaczuk W., Straszak J., Enigma: How the Poles Broke the Nazi Code, New York 2004.

L. Rivet, Carnets du chef des services secrets, 1936-1944, annotés par O. Forcade et S. Laurent, Paris 2010, p. 544-567.

Medrala J., Les réseaux de renseignements franco-polonais, Paris 2005.

Paillole P., Notre espion chez Hitler, Paris 2013.

Sebag-Montefiore H., Enigma: The Battle for the Code, Londres 2000.

Stengers J., Enigma, the French, the Poles and the British 1931-1940. Revue belge de philologie et d'histoire, tome 82, fasc. 1-2, 2004, p. 449-466.

Stripp A., A British salutes the Polish cryptanalysts [in :] W. Kozaczuk, J. Straszak, Enigma : How the Poles Broke the Nazi Code, New York 2004, p. 123-125.

Tyldum M., The Imitation Game, 2014.

Welchman G., The Hut Six story : Breaking the enigma codes, Londres 1997. 